\title{
Recurrent fourth-order interference dips and peaks with a comb-like two-photon entangled state
}

\author{
Alessandro Zavatta, Silvia Viciani, and Marco Bellin * \\ Istituto Nazionale di Ottica Applicata (INOA), \\ L.go E. Fermi, 6, I-50125, Florence, Italy
}

(Dated: November 1, 2018)

\begin{abstract}
We demonstrate full selective control over the constructive or destructive character of fourth-order recurrent interferences in a modified version of a Hong-Ou-Mandel interferometer using comb-like two-photon states. The comb spectral/temporal structure is obtained by inserting an etalon cavity in the signal path of an entangled photon pair obtained by pulsed spontaneous parametric downconversion. Both a simple qualitative discussion and a complete theoretical derivation are used to explain and analyze the experimental data.

PACS numbers: 42.50.Dv, 03.65.Ud
\end{abstract}

In the original scheme by Hong, Ou, and Mandel (HOM) 1] two single photons from a spontaneous parametric down-conversion (SPDC) pair are sent to two input ports of a beam-splitter (BS) and coincidences are observed between the detection events on two detectors placed at the BS output ports. While varying the relative phase delay between the two beams, one can observe a dip in the coincidence signal when such a delay is shorter than the coherence time of the downconverted photons. This fourth-order interference effect takes place when two photons in the same mode arrive simultaneously at BS and can also be observed when they are emitted independently by a single-photon device [2]. However, when an entangled two-photon state is considered, it is the indistinguishability between two two-photon amplitudes leading to the same detector "firing scheme" that gives rise to fourth-order interferences, and this may happen even if the two single photons arrive at BS at two different times (with a delay which can be much longer than their coherence time) and follow two distinguishable optical paths to reach the detectors. The interference will be either destructive or constructive, resulting in a dip or a peak in the coincidence signal, depending on the phase difference between these two two-photon amplitudes, as first observed by the group of Shih [3, 4, [5, 6].

Here, by using comb-like entangled states in a modified version of the HOM setup, we are able to force the concurrent contribution of both (HOM- and Shih-type) kinds of interferences to the generation of finely controllable dips or peaks in a recurrent pattern. A variable delay line is inserted in the idler path while an etalon cavity is placed in the other beam path and modifies the temporal structure of the signal photon wavepacket. The two photon wavepackets are then mixed at BS and coincidence events between detectors $\mathrm{D}_{1}$ and $\mathrm{D}_{2}$ at its output ports are measured while scanning the delay line (see Fig 1 for a simplified scheme of the experiment). We demonstrate a full control over the constructive or destructive character of the recurrent fourth-order interferences by means of a tuning of the etalon cavity in an easy and simply

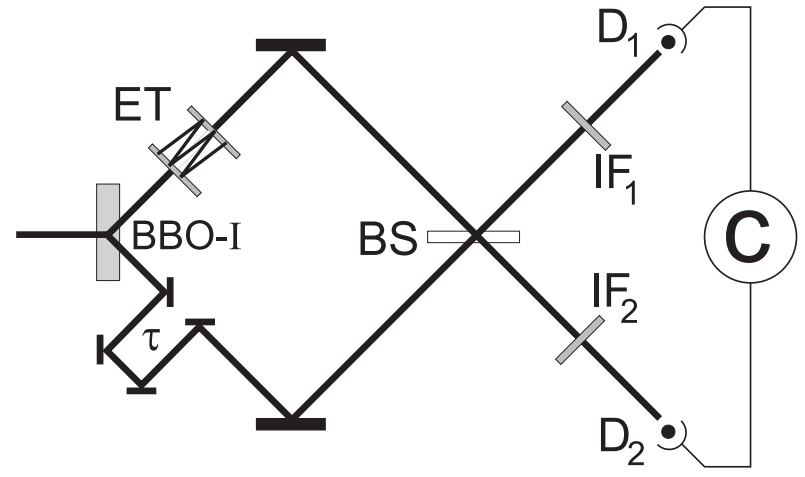

FIG. 1: Schematic view of the experimental set-up. See the text for details.

predictable way. This type of measurement has been recently discussed in a theoretical paper by Peřina [7] and a somewhat related experiment with so-called mode-locked two-photon states has also been recently reported by $\mathrm{Lu}$ et al. [8].

The etalon cavity is characterized by a comb-like spectral transmission function, with peaks equally spaced by the free-spectral-range (FSR), corresponding to the inverse of the cavity round-trip time $T(1 / F S R=T=$ $2 d / c$, where $d$ is the mirror separation and $c$ the speed of light). If the bandwidth of the down-converted photons is larger than the cavity FSR, several transmission modes of the resonator are simultaneously excited by the incoming field, and the output signal spectrum acquires a comb-like structure made of different peaks. It is also interesting to look at the effects of the etalon in the temporal domain: if the temporal coherence of the signal photon is much shorter than the etalon round-trip time, a signal photon wavepacket cannot interfere with its leading edge having been reflected twice by the mirrors inside the cavity. The result is a train of isolated coherent wavepackets of exponentially decreasing amplitude leaving the etalon equally spaced by the cavity round-trip time $T$. In general, the phase delay between consecutive pulses can be expressed 
as $\Delta \varphi=2 \pi n+\delta \varphi$, where $n$ is a positive integer and only the term $\delta \varphi$ plays a physically significant role. Note that the phase delay $\delta \varphi$ can be easily varied in a controllable way by slightly rotating the etalon along an axis perpendicular to the beam propagation direction, thus changing the optical path of the beam inside the cavity.

Now let us consider what happens when a single signal photon enters the etalon: in some cases it may be transmitted by both cavity mirrors and arrive to BS with just a small delay connected with the crossing of the mirror material; this is equivalent to the HOM case, and a dip in the coincidence rate is expected when the delay in the idler path is properly adjusted. Let us define this delay as $\tau=0$. Interference arises from the indistinguishability of the two possibilities for photons from the two paths of being both either transmitted or reflected by BS. If the mirror reflectivity is high enough, it is however more probable for the pulse to oscillate a few times inside the cavity before going out towards BS after a delay $m T$ corresponding to an integer number $m$ of roundtrips. If one just thinks of interference as arising from the temporal overlap of two one-photon wavepackets on the beamsplitter, then one might expect to see partial revivals of the interference dip also for idler path delays $\tau_{m}=m T$. Again, from a coincident detection event on the two detectors one cannot tell, not even in principle, if the photons have been both reflected or both transmitted by BS (see Fig 2 2 )). For each $\tau_{m}$, both these alternatives contribute with the same phase term, which thus factors out, leaving with the usual HOM state and with the appearance of a dip. According to this intuitive approach, only dips for delays $\tau_{m}$ equal to integer multiples of the cavity round-trip time $T$ are then to be expected. However, other indistinguishable alternatives leading to the same detector firing schemes (not necessarily coincidences!) are possible, and may thus lead to additional quantum interferences.

If the idler delay line is set such that $\tau=T / 2$ two firing configurations are possible, both of them allowing for two indistinguishable realizations represented by the Feynman-like diagrams of Fig 2 b) and c). In the first one (see Fig 2b)), detector $\mathrm{D}_{1}$ fires ahead of detector $\mathrm{D}_{2}$ by a time $T / 2$; this detection event can be realized either by letting the signal photon pass without reflections through the etalon and having both photons reflected by BS, or with one signal round-trip in the etalon and two transmissions through BS. In the second firing scheme, detector $\mathrm{D}_{2}$ clicks ahead of detector $\mathrm{D}_{1}$ by the same amount of time, as shown in Fig (2)): it can be realized either by a single signal round-trip through the etalon and two reflections on BS, or by transmission through the etalon and transmission of both photons through BS. In both these firing schemes the two interfering probability amplitudes differ by a phase term $\delta \varphi$ corresponding to one round-trip in the cavity. If $\delta \varphi$ is set to zero by an appropriate tilt of the etalon, the phase term cancels and a dip appears, a)

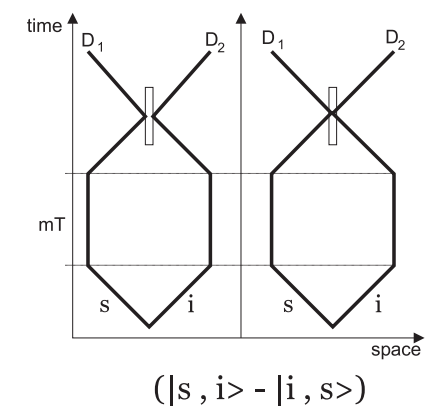

b)
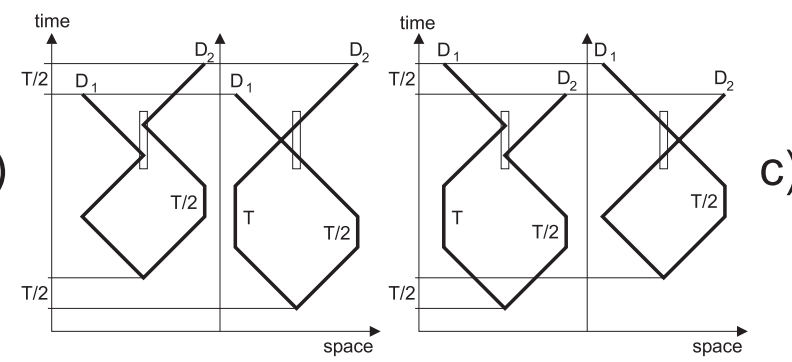

$$
\left(\left|s, i>_{0}-e^{i \delta \phi}\right| i, s>_{0}\right)+\left(\left|s, i>_{1}-e^{-i \delta \phi}\right| i, s>_{1}\right)
$$

FIG. 2: a): Feynman-like diagrams for the intuitive case where the idler delay is set equal to an integer number of etalon round-trips and photons arrive simultaneously at BS (HOM case). b) and c): The two pairs of interfering amplitudes for an idler delay corresponding to half a cavity roundtrip; the two photons reach BS at different times (Shih case).

but if $\delta \varphi=\pi$ then the dip becomes a peak while, for $\delta \varphi=\pi / 2$, the coincidence rate flattens out. Note that the two alternatives in both the above firing schemes imply a different time of birth of the photon pair inside the crystal, and are truly indistinguishable only if the difference between the times of generation $(T / 2$ in this case) is smaller than the pump coherence time. Although this is always true for a CW pump, it may constitute an important limitation to the visibility of interferences in the case of a pulsed pump with a short coherence time.

The above discussion can be generalized to the case where the idler photon delay is an integer multiple $j$ of half the round-trip time (i.e. $\tau_{j}=j T / 2$ ). If $m$ is the number of round-trips of the signal photon inside the etalon, one easily finds that, for each $j$, there are $j+1$ (corresponding to $m$ going from 0 to $j$ ) firing schemes possible, each allowing for two indistinguishable realizations. Each firing scheme (denoted by $m$ and illustrated in Fig 3) is the result of two alternative probability amplitudes, one involving $m$ and the other $(j-m)$ signal roundtrips, which present a phase difference of $(j-2 m) \delta \varphi$. Note that this phase difference is an even or odd multiple of $\delta \varphi$ depending on the parity of $j$.

In order to give a simple expression for the final comblike two-photon state and gain some insight into the terms contributing to these interference effects, we may 


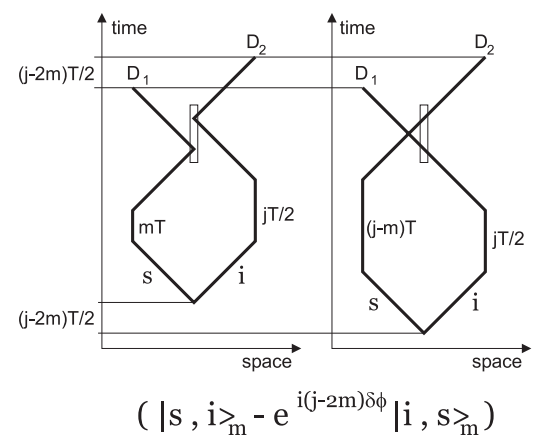

FIG. 3: General diagram for one of the $(j+1)$ terms contributing to quantum interferences in the coincidence counts when the idler delay is set to an integer number of half etalon round-trips: $\tau_{j}=j T / 2$.

make some crude approximations: we may assume that the pump is monochromatic (its coherence length is infinite) and that the reflectivity of the etalon mirrors is very high, so that we may neglect the amplitude decay between successive wavepackets exiting the cavity. The two-photon state at a given idler delay $\tau_{j}=j T / 2$, can then be written as:

$$
\left|\psi_{j}\right\rangle \propto \sum_{m=0}^{j}\left(|s, i\rangle_{m}-e^{i(j-2 m) \delta \varphi}|i, s\rangle_{m}\right)
$$

where the $|s, i\rangle_{m}\left(|i, s\rangle_{m}\right)$ term corresponds to the probability amplitude of a signal photon being detected at $D_{1}$ $\left(D_{2}\right)$ and an idler photon at $D_{2}\left(D_{1}\right)$ for a given firing scheme, and corresponds to the first (second) Feynmanlike diagram of Fig 3, For $\delta \varphi=0$, all the terms in eq1 contribute with a null phase, so that a sequence of dips is expected at idler delays corresponding to all $\tau_{j}=j T / 2$ (see Fig函).

For even $j$, one of the firing schemes (the one with $m=j / 2$ ) always corresponds to the HOM case described earlier and depicted in Fig 2 2 ). For $j=0$ this is the only possible contribution, so that for $\tau=0$ a dip is always present, independent of $\delta \varphi$. For $j>0$ however, there are additional (Shih-type) contributions which, depending on $\delta \varphi$, may be either constructive or destructive, so that the expected dip may, in some cases, flatten out or become a peak. Consider for example the case of $j=2$ (i.e. the idler delay is set at the cavity round-trip time $T)$ : as shown above, three contributions are present, one ( $m=1$, HOM-type) for which the phase term is zero and always contributes as a dip, and two others $(m=0,2$ Shih-type) with phase terms equal to $\pm 2 \delta \varphi$ which can be adjusted by rotating the etalon. If $\delta \varphi=\pi / 2$ (see

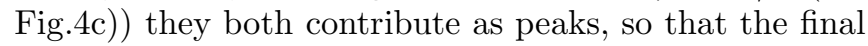
coincidence rate should show a small peak in this position. With this setting of the phase, it is also clear that all the delays corresponding to an odd $j$ (pure Shih-type interferences) will give a flat coincidence rate, being determined by contributions with phase terms which are odd multiples of $\pi / 2$. For the same reason, all these delay positions will change to peaks for $\delta \varphi=\pi$ (Fig $4 \mathrm{~b})$ ).

In this simplified situation, the behavior of the coincidences for different idler delays and for different settings of the etalon inter-pulse phase can be simply obtained by extending the above reasoning. Although useful for an intuitive understanding of the process and for a qualitative prediction of the experimental results, the above description is however too crude for a direct comparison of the measured data with calculations. A more refined and complete approach has then to be used, which takes into account all the parameters of the real experimental situation, like the finite coherence of the pump, the reflectivity of the etalon mirrors, the presence of spectral filters in the photon paths, and the natural spectral width of the SPDC two-photon state.

For low time-resolution detectors, the coincidence rate is obtained by a time integration of the second-order correlation function

$R_{c}=\int \mathrm{d} t_{1} \mathrm{~d} t_{2}\left\langle\psi\left|\hat{E}_{1}^{(-)}\left(t_{1}\right) \hat{E}_{2}^{(-)}\left(t_{2}\right) \hat{E}_{2}^{(+)}\left(t_{2}\right) \hat{E}_{1}^{(+)}\left(t_{1}\right)\right| \psi\right\rangle$.

The two-photon state generated by spontaneous parametric down-conversion in a $\chi^{(2)}$ nonlinear crystal is given by $[9,10]$

$$
|\psi\rangle=\int \mathrm{d} \omega_{s} \mathrm{~d} \omega_{i} \phi\left(\omega_{s}, \omega_{i}\right)\left|\omega_{s}\right\rangle_{s}\left|\omega_{i}\right\rangle_{i}
$$

where $|\omega\rangle_{s}$ and $|\omega\rangle_{i}$ are the single photon states for the signal and idler mode with frequency $\omega$, and the probability amplitude

$$
\phi\left(\omega_{s}, \omega_{i}\right)=\alpha \mathscr{E}_{p}^{(+)}\left(\omega_{s}+\omega_{i}\right) \frac{\sin (\Delta k L / 2)}{\Delta k L / 2} e^{-i \Delta k L / 2}
$$

for the two-photon state is the product of the pump envelope $\mathscr{E}_{p}^{(+)}(\omega)$ with the phase-matching function, which depends on the crystal phase-mismatch $\Delta k$ and on the crystal length $L$. The term $\alpha$ includes all the constants and the slowly varying terms. The field operators at the detectors are given by

$$
E_{1,2}^{(+)}(t)=\frac{1}{\sqrt{2}} \int \mathrm{d} \omega f(\omega)\left(\hat{a}_{s}(\omega) f_{e}(\omega) \pm \hat{a}_{i}(\omega) e^{i \omega \tau}\right) e^{i \omega t}
$$

where $\hat{a}_{s}(\omega)$, and $\hat{a}_{i}(\omega)$ are the annihilation operators for the signal and idler fields with frequency $\omega$ and $f(\omega)$ is the transmission function (assumed of Gaussian shape) of the interference filter placed in front of each detector. The etalon inserted in the idler beam is described by [1]

$$
f_{e}(\omega)=\frac{(1-R) e^{i \omega d / c}}{1-R e^{2 i \omega d \cos \theta / c}}
$$




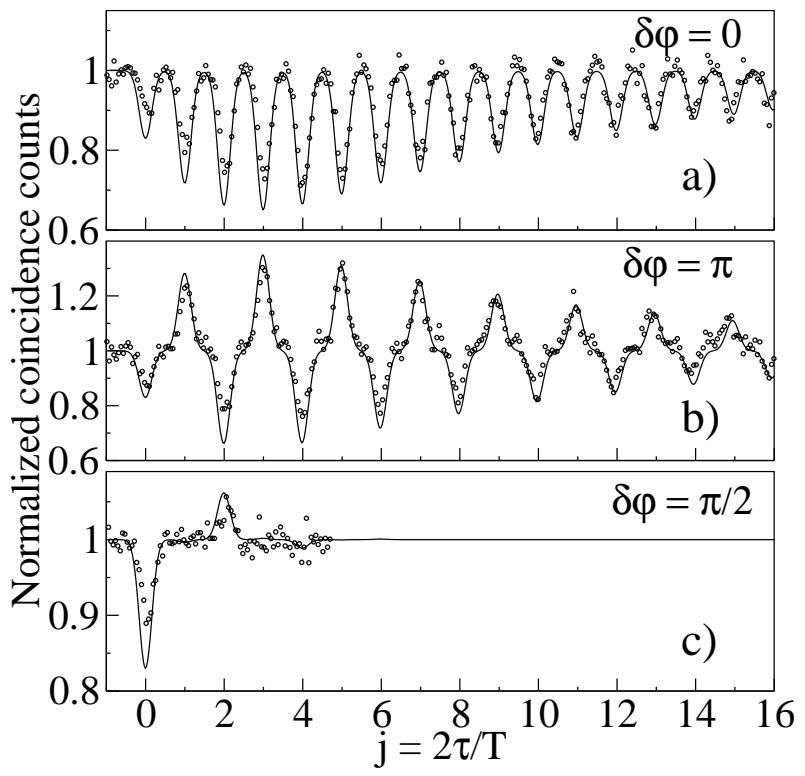

FIG. 4: Experimental results and calculated curves for the coincidence rates as a function of idler delay for three settings of the cavity inter-pulse phase delay.

where $d$ is the etalon mirror separation, $R$ the mirror reflectivity and $\theta$ the internal incidence angle. The coincidence rate is thus proportional to

$$
\begin{aligned}
& R_{c}(\tau)=\frac{1}{4} \int \mathrm{d} \omega_{i} \mathrm{~d} \omega_{s} F\left(\omega_{s}\right) F\left(\omega_{i}\right)\left\{\left|\phi\left(\omega_{s}, \omega_{i}\right)\right|^{2}\left|f_{e}\left(\omega_{s}\right)\right|^{2}\right. \\
& \left.-\phi\left(\omega_{s}, \omega_{i}\right) \phi^{*}\left(\omega_{i}, \omega_{s}\right) f_{e}\left(\omega_{s}\right) f_{e}^{*}\left(\omega_{i}\right) e^{-i\left(\omega_{s}-\omega_{i}\right) \tau}\right\}
\end{aligned}
$$

with $F(\omega)=|f(\omega)|^{2}$. When the etalon and interference filters are removed, the above expression is equivalent to that for the single HOM dip given in 12 .

The comb-like two-photon entangled state is experimentally generated by Type-I SPDC in a 3-mm long BBO crystal. The crystal is slightly tilted from the collinear condition in order to get degenerate parametric emission along a cone when pumped by the second harmonic of 1.4 ps-long pulses from a mode-locked Ti:sapphire laser operating around $786 \mathrm{~nm}$. The signal and idler photons are then selected by two apertures and sent to the two input ports of a $50 \%$ beam-splitter (BS), as schematically shown in Fig. 1] The air-spaced etalon placed on the path of the signal beam is made of a pair of plane and parallel facing mirrors with a reflectivity of $90 \%$ for a wavelength of $786 \mathrm{~nm}$, placed at a distance of $100 \mu \mathrm{m}$. The cavity FSR is $1500 \mathrm{GHz}$ (round-trip time $T=0.67 \mathrm{ps}$ ), corresponding to a width of about $3.1 \mathrm{~nm}$ at the central wavelength of the signal photon. The bandwidth of the downconverted photons is much larger than the etalon FSR and is anyway limited by the $10 \mathrm{~nm}$-wide interference filters (IFs) placed in front of the detectors. A motorized translation stage, placed on the path of the idler beam, is used to finely control the delay $\tau$ between the signal and idler photons. The beams emerging from the output ports of the BS are collected by means of $25 \mathrm{~mm}$ focal-length graded-index lenses, and detected by two single-photon counting modules, $\mathrm{D}_{1}$ and $\mathrm{D}_{2}$ (SPCM Perkin-Elmer AQR-12). Figure 4 shows the measured $\mathrm{D}_{1}-\mathrm{D}_{2}$ coincidence counts as a function of the idler delay for different settings of the inter-pulse phase $\delta \varphi$. Note that our 2 ns gate width also counts all the "delayed" coincidences depicted in Fig 3 as valid events. The condition $\delta \varphi=0$ is achieved by tilting the etalon so that one of its transmission maxima coincides with the interference filters' transmission peak. The phase delays $\delta \varphi=\pi$ and $\delta \varphi=\pi / 2$ are instead obtained by slight rotations of the cavity such that its comb-like spectrum is frequency shifted by FSR/2 and FSR/4, respectively. The solid curves in Fig. 4 show the result of the theoretical calculations according to eq. (2) without adjustable parameters. The agreement between the experimental and theoretical data is excellent and they both clearly confirm our previous qualitative discussion. The demonstration of an accurate control over the character of the individual interferences in the array produced by the comb-like entangled state may prove important for the implementation of new schemes in quantum state engineering and information processing.

This work has been supported by the Italian Ministry of University and Scientific Research (MIUR). The authors would also like to thank the Physics Dept. of the University of Florence for the kind hospitality and F. Marin for useful discussions.

* Also at LENS and INFM, Florence, Italy; Electronic address: bellini@inoa.it

[1] C. K. Hong, Z. Y. Ou, and L. Mandel, Phys. Rev. Lett. 59, 2044 (1987).

[2] C. Santori, D. Fattal, J. Vučković, G. S. Solomon, and Y. Yamamoto, Nature 419, 594 (2002).

[3] T. Pittman, D. V. Strekalov, A. Migdall, M. H. Rubin, A. V. Sergienko, and Y. H. Shih, Phys. Rev. Lett. 77, 1917 (1996).

[4] D. V. Strekalov, T. B. Pittman, and Y. H. Shih, Phys. Rev. A 57, 567 (1998).

[5] Y.-H. Kim, M. V. Chekhova, S. P. Kulik, and Y. Shih, Phys. Rev. A 60, 37 (1999).

[6] A. V. Burlakov, M. V. Chekhova, O. A. Karabutova, and S. P. Kulik, Phys. Rev. A 63, 053801 (2001).

[7] J. Peřina, Jr., Opt. Commun. 221, 153 (2003).

[8] Y. J. Lu, R. L. Campbell, and Z. Y. Ou, Phys. Rev. Lett. 91, 163602 (2003).

[9] Z. Y. Ou, Quantum Semiclass. Opt. 9, 599 (1997).

[10] T. E. Keller and M. H. Rubin, Phys. Rev. A 56, 1534 (1997).

[11] A. E. Siegman, Lasers (University Science Books, Mill Valley, California, 1986).

[12] W. P. Grice and I. A. Walmsley, Phys. Rev. A 56, 1627 (1997). 